\title{
The Continuing Need for Electron Microscopy in Examination of Medical Renal Biopsies: Examples in Practice
}

\author{
Michifumi Yamashita Mercury Y. Lin Jean Hou Kevin Y.M. Ren Mark Haas \\ Department of Pathology and Laboratory Medicine, Cedars-Sinai Medical Center, Los Angeles, CA, USA
}

\section{Keywords \\ Minimal change disease - Membranous nephropathy · Postinfectious glomerulonephritis - Alport syndrome . Collagen type III glomerulopathy}

\begin{abstract}
Background: For the better part of the past 6 decades, transmission electron microscopy (EM), together with routine light microscopy and immunofluorescence and/or immunohistochemistry (IHC), has been an essential component of the diagnostic workup of medical renal biopsies, particularly native renal biopsies, with increasing frequency in renal allograft biopsies as well. Studies performed prior to the year 2000 have indeed shown that a substantial fraction of renal biopsies cannot be accurately diagnosed without EM. Still, EM remains costly and labor-intensive, and with increasing pressure to reduce healthcare costs, some centers are deemphasizing diagnostic EM. This trend has been coupled with advances in $\mathrm{IHC}$ and other methods in renal biopsy diagnosis over the past 2-3 decades. Summary: Nonetheless, it has been our experience that the diagnostic value of EM in the comprehensive evaluation of renal biopsies remains similar to what it was 20-30 years ago. In this review, we provide
\end{abstract}

karger@karger.com www.karger.com/gdz

Karger $\stackrel{\text { ! }}{\div}$
(C) 2021 The Author(s)

Published by S. Karger AG, Basel

This is an Open Access article licensed under the Creative Common Attribution-NonCommercial-4.0 International License (CC BY-NC) (http://www.karger.com/Services/OpenAccessLicense), applicable to the online version of the article only. Usage and distribution for commercial purposes requires written permission. several key examples from our practice where EM was essential in making the correct renal biopsy diagnosis, ranging from relatively common glomerular lesions to rare diseases. Key Messages: EM remains an important component of the diagnostic evaluation of medical renal biopsies. Failure to perform EM in certain cases will result in an incorrect diagnosis, with possible clinical consequences. We strongly recommend that tissue for EM be taken and stored in an appropriate fixative and ultrastructural studies be performed for all native renal biopsies, as well as appropriate renal allograft biopsies as recommended by the Banff consortium.

\section{(c) 2021 The Author(s) \\ Published by S. Karger AG, Basel}

\section{Introduction}

Electron microscopy (EM), together with routine light microscopy (LM) and immunofluorescence (IF) and/or immunohistochemistry, has long been an essential component in the diagnostic workup of medical renal biopsies. Justification for routinely performing EM on native renal biopsy specimens was provided by a study we performed over 2 decades ago, which demonstrated that in 50/233 (21\%) consecutive biopsies, EM 
was necessary to make a correct diagnosis. In another 48 (21\%) cases, EM provided important contributory data, and in $8(3 \%)$ others, an additional diagnosis was supported by the ultrastructural findings [1]. Furthermore, the Banff group is now recommending an increased use of EM in examination of renal allograft biopsies, not only in cases of suspected recurrent or de novo glomerular diseases but also to identify early lesions of transplant glomerulopathy months to years before this becomes apparent by LM, possibly at a time point where treatment may prevent progression to overt transplant glomerulopathy $[2,3]$. Nonetheless, it is a fact that EM is a costly and labor-intensive technique, requiring not only very expensive equipment but also technologists with specialized training to cut and stain ultrathin tissue sections. With ever-increasing pressure worldwide to reduce healthcare costs, as well as recent advances in immunohistochemistry that have the potential to diagnose certain renal lesions previously identifiable only with the use of EM (e.g., [4-6]), some renal pathology laboratories are de-emphasizing the role of diagnostic EM. Despite this trend, it has been our experience that the value of EM in accurate renal biopsy diagnosis remains similar to what it was 20-30 years before. In this review, we provide key examples from our practice where EM was essential in making the correct renal biopsy diagnosis, ranging from relatively common glomerular lesions to rare diseases.

\section{Teaching Example \#1 - Minimal Change Nephropathy}

A 14-year-old male patient with a history of Hashimoto thyroiditis and chronic NSAID use presented with nephrotic syndrome and acute kidney injury (AKI). His serologic workup was unremarkable.

The tissue processed for LM contained 15 glomeruli, which all showed single contoured capillary walls without visible glomerular basement membrane (GBM) spikes. In a minority of glomeruli, mild segmental mesangial hypercellularity was noted. No segmental sclerosing lesions were present, and there was no endocapillary hypercellularity, glomerular necrosis, or crescent formation. Some proximal tubules displayed evidence of acute injury with epithelial flattening, diminished brush borders, and intraluminal sloughing of individual epithelial cells. There was no significant tubulointerstitial scarring or inflammation, and arteries and arterioles were unremarkable. IF studies, performed on a sample of renal cor- tex with 10 glomeruli, showed no glomerular staining other than trace to $1+$ granular mesangial staining for IgM. Tubular epithelial protein reabsorption droplets stained strongly for albumin and weakly for IgG, kappa, and lambda.

The tissue processed for EM contained 4 histologically unremarkable glomeruli, 3 of which were examined ultrastructurally. EM showed GBMs with normal thickness and trilaminar structure. There were rare, small mesangial electron-dense deposits; no subepithelial or subendothelial deposits or tubulo-reticular inclusions (TRIs) were noted. There was extensive, near-total podocyte foot process effacement with segmental microvillous transformation (Fig. 1a, b). No extraglomerular electron-dense deposits were present. Proximal tubules showed segmental flattening of the epithelium and attenuation of the brush border (Fig. 1c).

The diagnosis was minimal change disease (MCD) and mild acute tubular injury. The latter could be related to NSAID use, and the possibility of NSAID-induced MCD was raised in a comment. Acute tubular injury and AKI are common complications of MCD in older adults, particularly those with underlying hypertensive vascular disease, as a result of hemodynamic abnormalities [7]. Although this is a young, normotensive patient, the tubular injury could also be hemodynamically related in this case.

\section{Discussion}

MCD typically presents with nephrotic syndrome and is most common in young children, often younger than 6 years [8]. It is more common in males and in Caucasian and Asian children. Although less common, MCD can also present bimodally in older adults and has reported associations with malignancy, viral infections, allergies, and medication exposure. These adult cases of MCD also tend to have higher frequencies of hypertension, renal failure, and microscopic hematuria [9].

IF microscopy in MCD is typically negative in glomeruli, although some cases of MCD can be associated with modest mesangial IgM deposition. Whether this IgM is associated with a worse response to steroid therapy is controversial. Very early lesions of membranous nephropathy $(\mathrm{MN})$, where subepithelial GBM spikes have not yet formed, can mimic MCD by LM, and may show nondiagnostic IF findings. As such, rendering a diagnosis of MCD in nephrotic patients based purely on light microscopic findings, particularly in, but not limited to, cases where IF studies were not possible, is not advised without confirmatory EM. 
Fig. 1. Ultrastructural changes in MCD. a Extensive/near-complete effacement of podocyte foot processes. Glomerular capillary basement membranes have normal thickness and architectural organization. b Condensation of microfilaments (arrows) within podocyte at the epithelial surface of GBM. c Acute tubular injury with flattened epithelial lining and marked attenuation of proximal tubular brush border. d Partially treated MCD with partial restoration of podocyte foot processes. All electron micrographs stained with uranyl acetate and lead citrate; original magnifications $\times 10,000(\mathbf{a}), \times 19,000(\mathbf{b}), \times 5,800$ (c), $\times 10,000$ (d). GBM, glomerular basement membrane; MCD, minimal change disease.
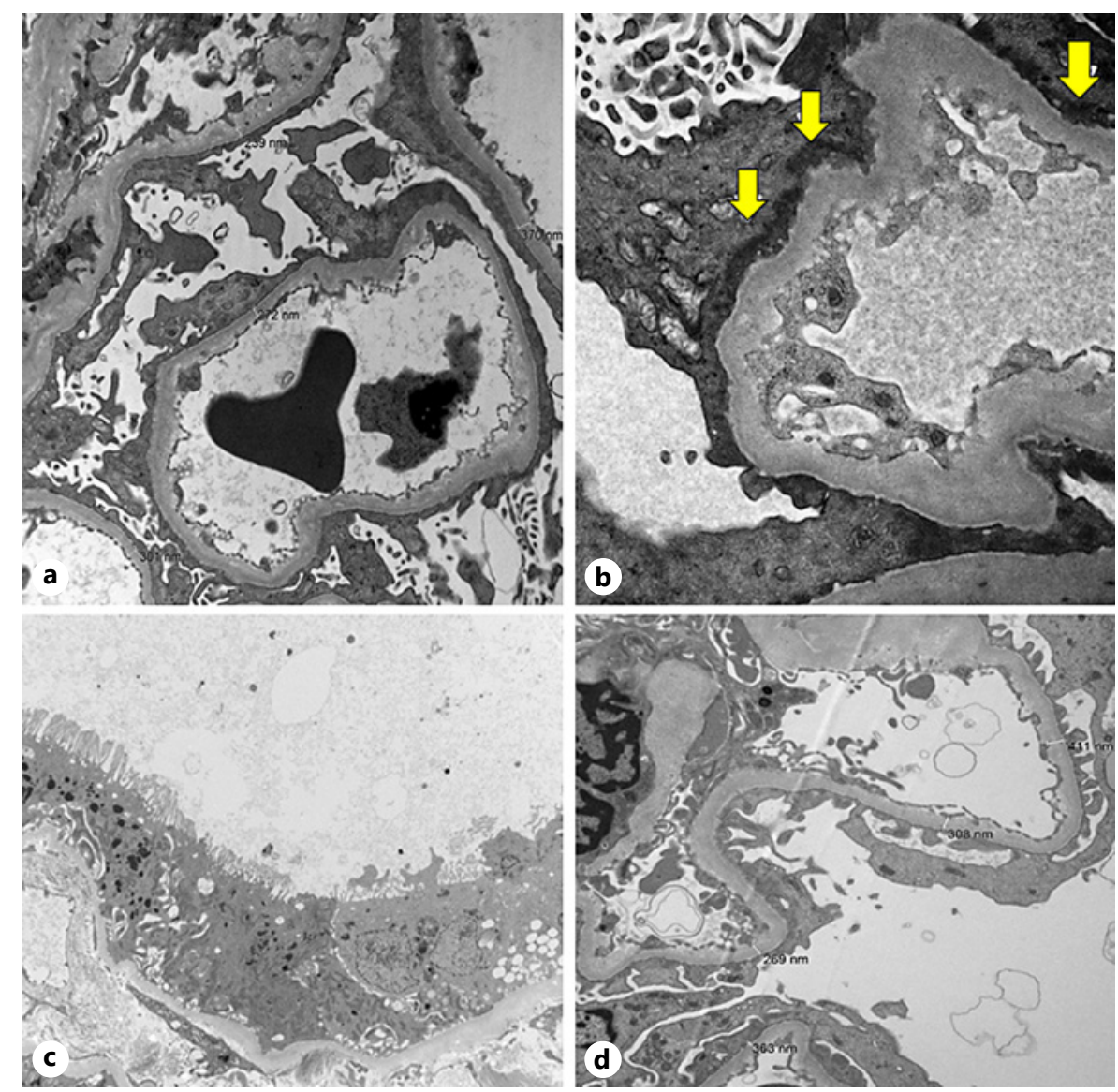

By EM, the hallmark of MCD is extensive and complete or nearly complete effacement of podocyte foot processes [10]. For accurate evaluation of the extent of the foot process effacement, only well-preserved, patent (nonischemic) glomeruli should be selected for ultrastructural examination. An additional confounding factor to consider is the possibility of therapeutic effect. Pediatric patients with MCD are typically biopsied only after treatment failure or incomplete response, and in this setting, the extent of podocyte foot process effacement may not be as extensive (e.g., Fig. 1d as compared to Fig. 1a). As such, an appropriate clinical history is required to establish a diagnosis of partially treated MCD or MCD in which there has been a spontaneous remission of proteinuria. In MCD, there are no glomerular capillary wall deposits. Nonetheless, it should be noted that condensation of podocyte microfilaments can be mistaken for subepithelial immune deposits of early $\mathrm{MN}$ on lower power electron micrographs. However, as shown in Figure $1 \mathrm{~b}$, the cytoskeletal nature of the condensed material can be discerned at higher power.

Electron Microscopy in Kidney Pathology

\section{Teaching Example \#2 - Early MN}

A 59-year-old female patient with a history of hypertension and prediabetes presented with proteinuria of approximately 5 grams per day. She had a serum creatinine of $0.8 \mathrm{mg} / \mathrm{dL}$, low serum albumin of $3.2 \mathrm{~g} / \mathrm{dL}$, weakly positive ANA, but normal serum $\mathrm{C} 3$ and $\mathrm{C} 4$. She was negative for ANCA, hepatitis B and C, and HIV. There was no family history of renal disease.

The specimen for LM contained 39 glomeruli, one of which was globally sclerotic. The remaining glomeruli were of normal size and cellularity with normal appearance of capillary loops, and there were no distinct GBM double contours, spikes, or craters on silver stain. No glomeruli showed segmental sclerosis, crescents, or endocapillary hypercellularity, and the mesangial areas were unremarkable. The non-atrophic tubules were of normal size without epithelial flattening or loss of brush borders. The renal cortex showed approximately $15-20 \%$ tubular atrophy and interstitial fibrosis with focal mild lymphocytic inflammation in areas of scarring. Arteries showed 
Fig. 2. Findings in early MN. a Direct IF for IgG. Glomerular capillary walls show segmental granular to linear staining for IgG $(1+$ on $0-4+$ scale). Mesangial areas are negative (fluorescein-conjugated anti-human IgG, original magnification, $\times 400$ ). b EM shows several subepithelial electrondense (immune complex) deposits (arrows). Overlying podocytes show extensive foot process effacement (uranyl acetate and lead citrate stain; original magnification, $\times 4,800$ ). c A higher magnification of the same capillary shows minimal to very early extension of the GBM (arrows) around the deposits (original magnification, $\times 10,000$ ). d IHC for THSD7A shows weak staining of podocyte cell bodies and moderate staining in glomerular capillary wall deposits (original magnification, $\times 400$ ). EM, electron microscopy; IF, immunofluorescence; GBM, glomerular basement membrane; MN, membranous nephropathy; THSD7A, thrombospondin type 1 domaincontaining 7A; IHC, immunohistochemistry.
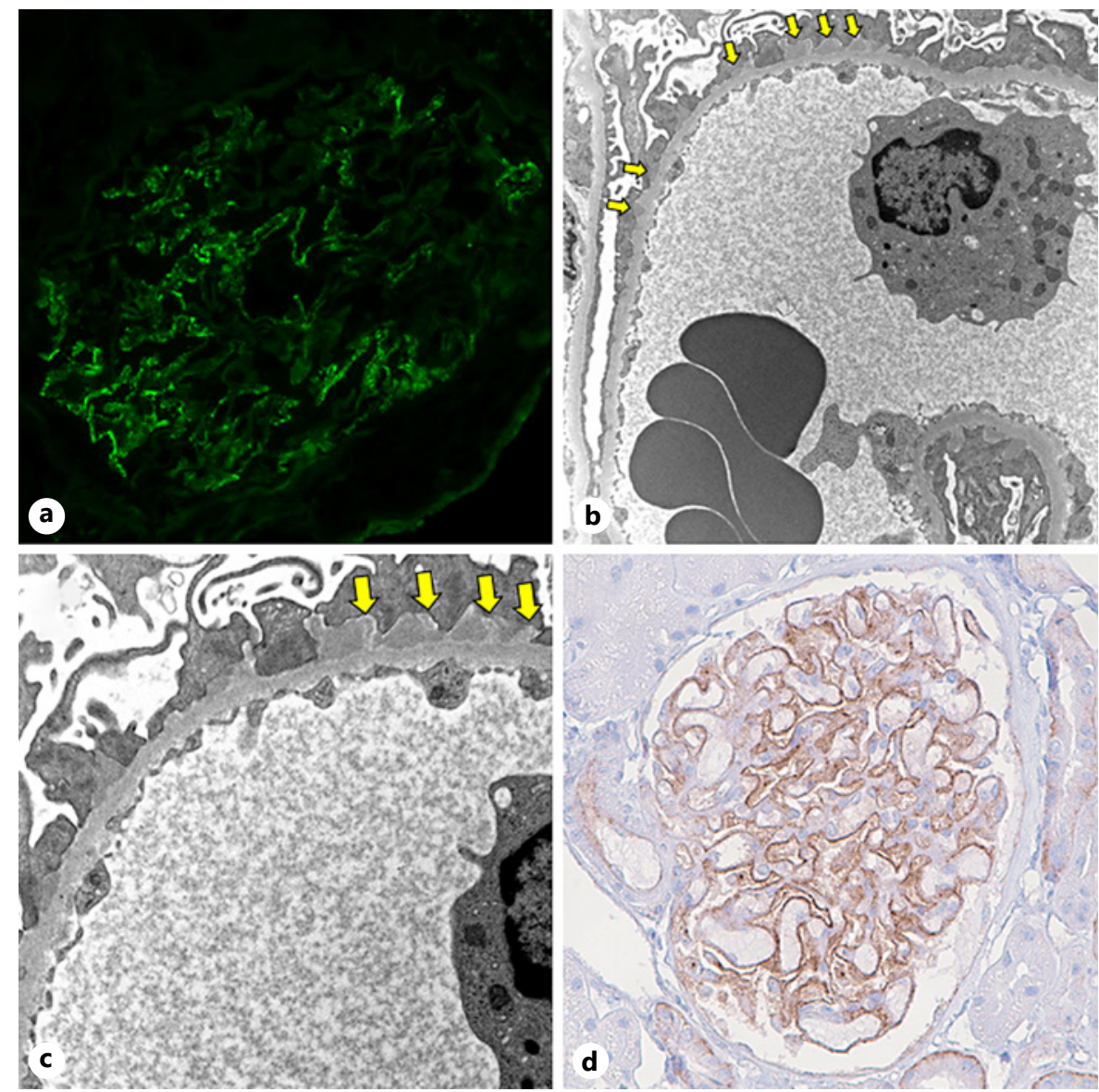

mild intimal fibrosis and thickening. No active arteritis was noted. Arterioles showed focal mild hyalinosis.

IF studies showed segmental, equivocal linear to finely granular staining for IgG (1+) (Fig. 2a), kappa (trace to $1+$ ), and lambda (trace to $1+$ ) light chains along glomerular capillary walls. There was no glomerular staining for IgA, IgM, C1q, or C3.

EM performed on 3 glomeruli without segmental lesions showed segmental subepithelial electron-dense immune complex deposits (Fig. 2b, c). Some glomerular capillaries had few or no deposits, while others had many. Some deposits showed very early extension of the GBMs around the deposits; however, most of the deposits simply appeared to rest on the GBMs. The GBMs themselves showed a normal trilaminar structure and a mean thickness within the normal range. No mesangial deposits, subendothelial deposits, or tubular basement membrane deposits were noted. The glomerular endothelial cells were unremarkable without TRIs. There was moderately extensive (estimated approximately 70\%) podocyte foot process effacement without microvillous transformation. Foot process effacement was greatest in those glomerular capillaries with large number of deposits.

Based on the EM findings, immunohistochemical studies for phospholipase A2 receptor (PLA2R) [4] and thrombospondin type 1 domain-containing 7A (THSD7A) [5] were performed. PLA2R was negative in the glomerular capillary walls, while THSD7A showed diffuse, moderate glomerular capillary wall staining with a finely granular appearance (Fig. 2d). There was some staining of podocyte cell bodies for THSD7A; however, this was clearly weaker than the capillary wall staining. The final renal biopsy diagnosis was early $\mathrm{MN}$, predominantly stage I of Ehrenreich and Churg [11], positive for THSD7A.

\section{Discussion}

$\mathrm{MN}$ is an autoimmune glomerular disease characterized by subepithelial immune complex deposits with nephrotic range proteinuria; this may be associated with an 
Fig. 3. Case of old/resolved MN, stage IV. a GBMs show numerous craters (Jones' silver methenamine stain; original magnification, $\times 600$ ). b On EM, GBMs shows many largely reabsorbed intramembranous deposits (arrows), giving the GBMs a "moth-eaten" appearance (uranyl acetate and lead citrate, original magnification, $\times 7,200)$. EM, electron microscopy; GBMs, glomerular basement membranes; $\mathrm{MN}$, membranous nephropathy.

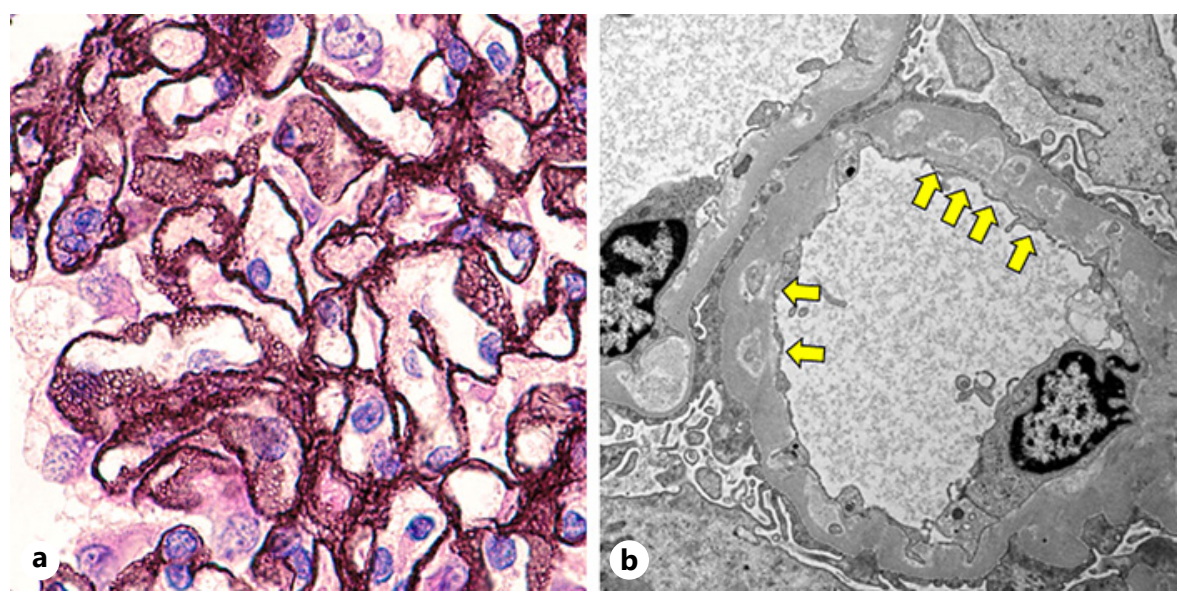

Table 1. Comparison of EM features of early MN and MCD

\begin{tabular}{ll}
\hline Early MN & MCD \\
\hline $\begin{array}{l}\text { Subepithelial electron-dense (immune complex) deposits } \\
\text { Podocyte foot process effacement mainly in areas overlying the subepithelial deposits } \\
\text { Subendothelial and/or mesangial deposits if secondary MN, e.g., membranous lupus nephritis [12] }\end{array}$ & $\begin{array}{l}\text { No electron-dense deposits } \\
\text { Complete or nearly complete podocyte foot process } \\
\text { TRIs in endothelial cells in membranous lupus nephritis [12] } \\
\text { Often microvillous transformation in podocyte cell } \\
\text { bodies }\end{array}$
\end{tabular}

EM, electron microscopy; TRIs, tubuloreticular inclusions; MCD, minimal change disease; MN, membranous nephropathy.

underlying systemic disease such as lupus [12], with viral infections including hepatitis B and C $[13,14]$, with an underlying neoplasm $[15,16]$ or with administration of therapeutic agents $[15,16]$ (secondary $\mathrm{MN}$ ), or may be primary. As approximately, 70\% of cases of primary $\mathrm{MN}$ result from autoantibodies against PLA2R [4], whereas $3-5 \%$ are due to antibodies against THSD7A [5], the term primary $\mathrm{MN}$ is preferable to idiopathic $\mathrm{MN}$ to describe these cases. Well-established MN is easily identifiable by LM; however, very early (stage I of Ehrenreich and Churg [11]) MN typically shows histologically unremarkable glomeruli. Furthermore, IF staining for IgG in very early $\mathrm{MN}$ may be equivocal (sometimes even more so than in Fig. 2a), and thus, by LM and IF such lesions may be indistinguishable from MCD, which manifests a similar clinical picture. As such, this distinction must be made by EM; a comparison of ultrastructural features of early MN and MCD is shown in Table 1.

Cases of old/resolved MN (stage IV of Ehrenreich and Churg [11]) may also prove challenging to make a diagnosis based only on LM and IF. While LM may show GBM craters, depressions in the GBM seen in en face sec-

Electron Microscopy in Kidney Pathology tions on silver stain (Fig. 3a), these are not always easily identifiable, and IF often shows equivocal or no glomerular IgG staining. For such cases, EM is the key study for the precise pathological diagnosis and reveals numerous largely reabsorbed intramembranous deposits (Fig. 3b).

\section{Teaching Example \#3 - Alport Syndrome}

A 17-year-old white male patient presented with nephrotic syndrome, having first reported lower extremity edema 4 months earlier. Serum creatinine was $1.15 \mathrm{mg} /$ $\mathrm{dL}$ with a urine protein/creatinine ratio of 7.5. Urinalysis showed $6-10$ red blood cells and $0-2$ white blood cells per high-power field. He was normotensive and reported no symptoms other than edema. Hepatitis B and C, ANA, and HIV were negative, and serum $\mathrm{C} 3$ and $\mathrm{C} 4$ were within the normal range. He reported no family history of renal disease.

The tissue processed for LM contained 17 glomeruli, 3 globally sclerotic. The remaining glomeruli showed no hypercellularity, crescents, or necrotizing lesions. Two 

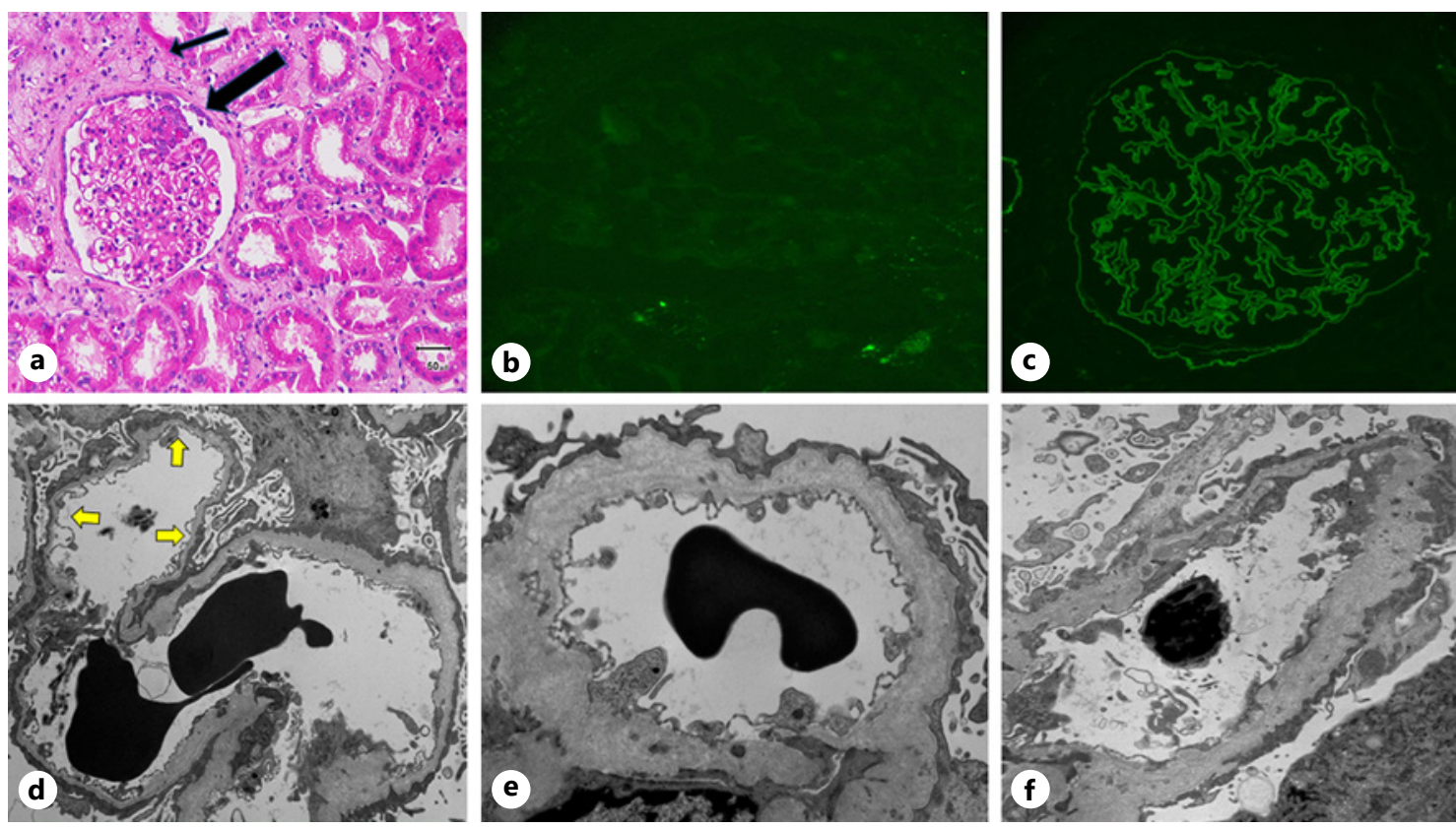

Fig. 4. LM, (IF, and EM findings in a case of X-linked AS. a LM shows a normocellular glomerulus with segmental sclerosis and a "cap" of epithelial cells overlying the sclerotic segment (thick arrow). Small aggregates of interstitial foam cells (thin arrow) were also noted (hematoxylin and eosin stain, original magnification, $\times 200$ ). $\mathbf{b}, \mathbf{c}$ Indirect IF for the alpha- 5 chain of type IV collagen is completely negative (b), while a normal control kidney showed diffuse linear staining in GBMs and Bowman's capsule, and focal linear tubular basement membrane staining (c), the latter at left (original magnification of both micrographs $\times 400$ ). $\mathbf{d}-\mathbf{f}$ EM shows highly variable GBM thickness with thick and thin segments (ar- rows) (d, f), with irregular, scalloped outer contours of the GBM, the latter most evident in areas of GBM thickening (e). GBM splitting and lamellation is also most evident in the thickened areas (e), and occasional electron-dense microparticles are seen within the GBM (f). In panel f, GBM thinning and thickening with splitting/ lamellation are seen in the same glomerular capillary. Podocyte foot processes show extensive, almost complete effacement (uranyl acetate and lead citrate stain; original magnifications, $\times 7,200$ (d), $\times 14,000$ (e), $\times 7,200$ (f)). EM, electron microscopy; LM, light microscopy; IF, immunofluorescence; GBM, glomerular basement membrane; AS, Alport syndrome. glomeruli showed segmental sclerosis; in one, this was very segmental with a small "cap" of epithelial cells overlying the sclerotic segment (Fig. 4a), while in the other, the sclerosis was more advanced, involving approximately half of the glomerular tuft. There was mild interstitial fibrosis and tubular atrophy (estimated approximately $10-15 \%)$ with very little interstitial inflammation, although occasional small clusters of interstitial foam cells were noted (Fig. 4a). Arteries and arterioles were unremarkable.

Direct IF studies showed no glomerular deposits of immunoglobulins or complement components. A preliminary diagnosis of focal segmental glomerulosclerosis (FSGS), most likely primary, given the patient's age and clinical history, was made at this time.

EM was performed on a single glomerulus which was normocellular without segmental lesions on one-micronthick sections of the plastic embedded tissue. As shown in
Figure 4, panels D-F, there was marked variability in the thickness of the GBMs, ranging from very thin $(<100 \mathrm{~nm})$ to very thick $(>1,000 \mathrm{~nm})$, with normal thickness for the patient's age and gender being $330 \pm 50$ (SD) $\mathrm{nm}$ at our center. Most notable in the thickened areas the GBMs had a lamellated or "basket weave" structure without a distinct lamina densa. There were also markedly irregular and scalloped or "sawtooth-like" outer (epithelial) contours of the GBMs; the latter 2 features are best appreciated in Figure 4e. No glomerular or extraglomerular immune complex deposits were seen, although segmentally the GBM contained some small intramembranous electron-dense granules or microparticles (Fig. 4f). Podocyte foot processes were almost completely effaced. No structural abnormalities of tubular basement membranes were seen.

Because of the EM findings, additional IF studies for alpha- 3 and alpha- 5 chains of type IV collagen were per- 
formed. These showed a complete absence of staining in GBMs, Bowman's capsules, and distal tubular basement membranes for either alpha-3 (IV) or alpha-5 (IV) (Fig. 4b). By contrast, linear staining of all 3 structures was seen in a normal control kidney (Fig. 4c).

The final renal biopsy diagnosis was thus Alport syndrome (AS), X-linked type, with secondary focal and segmental glomerulosclerosis. Initially, there was no reason to suspect AS based on the clinical history, LM, and routine IF studies. While the patient did have microscopic hematuria, this is not infrequently seen with FSGS, and the patient did not report a family history of renal disease or hearing loss. Furthermore, while there were some interstitial foam cells noted histologically, these are not specific and may been seen in in the context of proteinuria of various etiologies, especially if present for a significant time interval. In this case, the GBM abnormalities identified by EM were absolutely crucial for the proper diagnosis of AS. There are several clinical implications of this diagnosis for the patient, including likely sparing him from immunosuppressive therapy, such as high-dose corticosteroids. In addition, the diagnosis will likely lead to a closer screening of his family members, and the patient should plan appropriate genetic counseling to have children.

\section{Discussion}

AS and thin GBM nephropathy (TBMN) are characterized by GBM ultrastructural abnormalities. Although both conditions typically present with hematuria, AS is more commonly associated with progressive decline in renal function, proteinuria, and development of extra-renal manifestations including sensorineural hearing loss and ocular lesions $[17,18]$. The genetic bases for both conditions are heterogeneous and usually involve mutations in gene(s) coding for various chains of type IV collagen [19].

Approximately $85-90 \%$ of AS cases are X-linked and result from mutations in the COL4A5 gene, located on the $\mathrm{X}$ chromosome, coding for the $\alpha_{5}$ subunit of type IV collagen $[17,18]$. The remaining cases of AS are secondary to mutations in COL4A3 and COL4A4 coding for the $a_{3}$ or $\alpha_{4}$ subunits of type IV collagen, respectively, with autosomal recessive AS accounting for approximately 10 $15 \%$ of the cases and autosomal dominant AS making up a small number [19]. Approximately, $40 \%$ of TBMN patients have identifiable heterozygous mutations in $\mathrm{CO}$ $L 4 A 3$ or COL $4 A 4$ loci and may also be considered carriers of autosomal recessive AS [19]. While indirect IF for type IV collagen $\alpha_{3}$ and $\alpha_{5}$ chains can be used to confirm the loss of expression of one or both of these chains in AS and to distinguish between the different types of AS based on the staining pattern $[17,18]$, some cases of AS with intact staining for $\alpha_{3}$ (IV) and $\alpha_{5}$ (IV) have been described [6]. As such, AS cannot be ruled out solely by IF. EM is key to making the diagnoses of AS and TBMN, and without EM, many such cases would be misdiagnosed, including the case described above.

The prototypical ultrastructural lesions of AS are illustrated in Figure 1d-f and are characterized by irregular GBMs with alternating segments of thickening and thinning, lamellation and splitting ("basket-weave" appearance) of the lamina densa with loss of normal trilaminar GBM architecture, small electron-dense granules or microparticles within the GBM, and an irregular, scalloped outer (epithelial) contour of the GBM $[17,18]$. It should be noted that all of the above ultrastructural features are not always observed in individual cases of AS, and significant ultrastructural variation may exist between AS patients.

GBM splitting and lamellation may be quite patchy early in the disease course but typically becomes more extensive with disease progression on serial biopsies [1921]. Diffuse GBM thinning similar to that seen in TBMN can predominate in younger patients with early stages of AS or in female carriers of X-linked AS [22]. However, GBM abnormalities more characteristic of AS may develop early in life, and close proximity of thin and thick, split and lamellated GBM may be present within the same capillary in children and young patients (Fig. 1f). GBM thickening and diffuse lamellation and splitting may predominate in advanced AS, particularly in male patients with X-linked AS, and can be associated with certain families and can indicate severe disease, while some other patients have only diffuse GBM thinning, regardless of the age or type of COL4 mutation [19, 23, 24].

GBM irregularities and variable thickness are not specific for AS and can be observed in hyperfiltration injury as well as other glomerular diseases including FSGS, advanced $\mathrm{MN}$, postinfectious glomerulonephritis (PIGN), or other types of immune complex-mediated glomerulonephritis (GN) with largely resorbed deposits and significant GBM remodeling $[17,25,26]$. Widespread GBM abnormalities mimicking those seen in AS have also been described in rare pediatric cases of steroid-resistant nephrotic syndrome, Denys-Drash or Frasier syndrome with WT1 mutation, Pierson syndrome with LAMB2 mutation, and nephrotic syndrome associated with hypomelanosis of Ito without known COL4 mutations [19, $27-29]$. 
Fig. 5. IgA-dominant PIGN. a There are mesangial and segmental endocapillary hypercellularity, with a segmental, predominantly cellular crescent (periodic acidSchiff stain, original magnification, $\times 400$ ). b IF for IgA shows granular staining in the mesangium with segmental staining in glomerular capillary walls, the latter very segmentally with a coarsely granular appearance (fluorescein isothiocyanate-conjugated anti-human IgA, original magnification, $\times 200)$. c EM shows smudgy subendothelial (arrow) and mesangial deposits (arrowhead). Note the intracapillary leukocytes (uranyl acetate and lead citrate, original magnification, $\times 7,500)$. d Segmentally prominent subepithelial "hump"-shaped deposits along a peripheral capillary loop, with scattered smaller intramembranous and subendothelial deposits (uranyl acetate and lead citrate, original magnification, $\times 12,000)$. EM, electron microscopy; IF, immunofluorescence; PIGN, postinfectious glomerulonephritis.
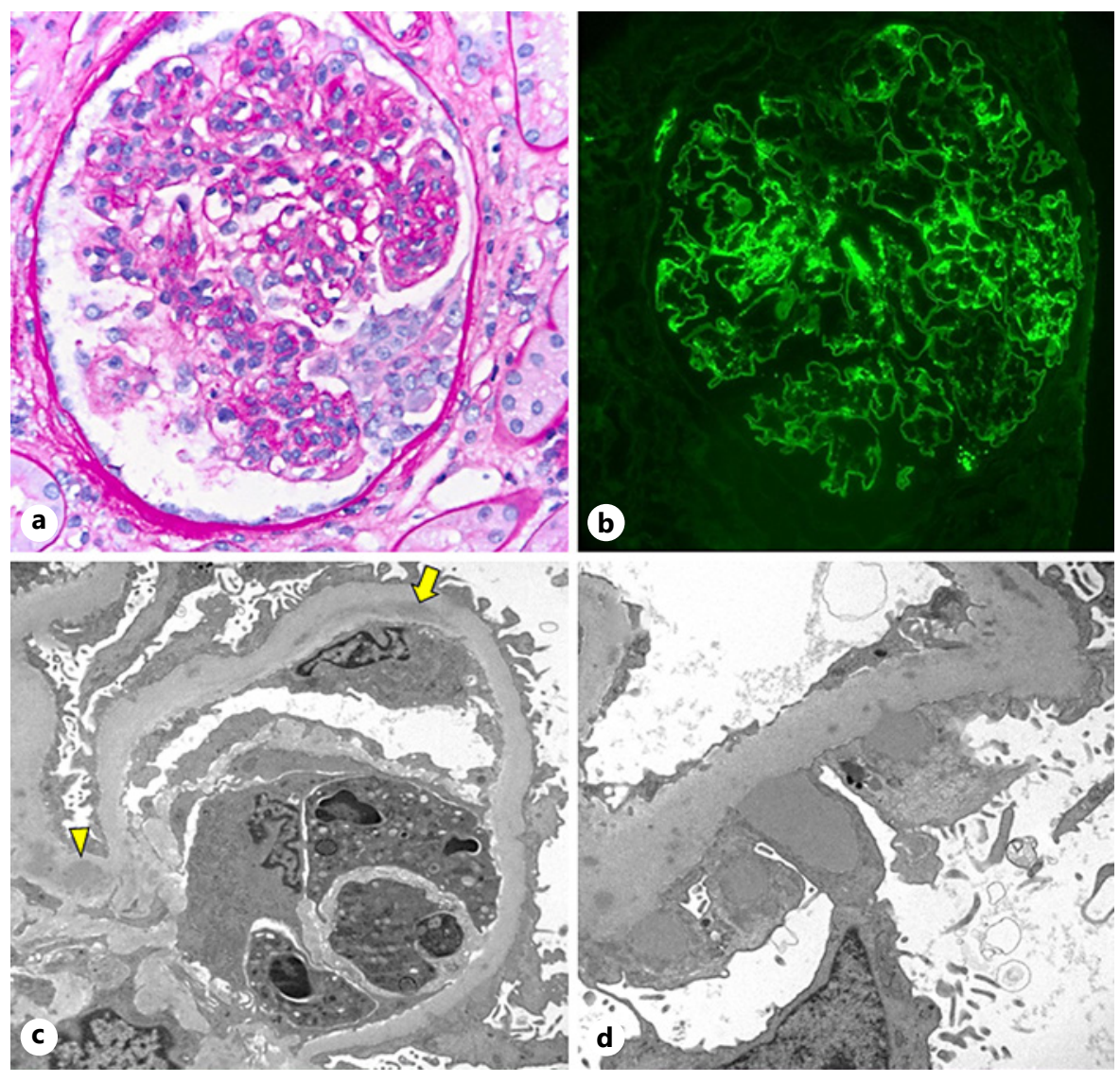

\section{Teaching Example \#4 - IgA-Dominant PIGN (Infection-Related)}

An 80-year-old man with type 2 diabetes presented with sub-nephrotic range proteinuria, moderate microscopic hematuria, and AKI. Serologic workup including hepatitis B and C and ANCA was negative, serum C3 and $\mathrm{C} 4$ were within the normal range, and there was no evidence of a monoclonal paraprotein. There was no known history of a recent infection.

LM showed 10 glomeruli, none of which were globally sclerotic. Glomeruli showed mesangial and segmental endocapillary hypercellularity; one glomerulus showed a segmental, predominantly cellular crescent (Fig. 5a). No glomerular necrotizing lesions were seen, although one glomerulus contained a moderate number of neutrophils. There were rare GBM double contours. On the trichrome stain, mesangial areas showed scattered fuchsinophilic deposits; however, no definitive subepithelial "hump"shaped deposits were seen. None of the glomeruli showed nodular glomerulosclerosis. There were only mild tubu- lar atrophy and interstitial fibrosis, and mild arterio- and arteriolosclerosis.

By IF, glomeruli displayed global mesangial and segmental capillary wall staining for IgA (3-4+; Fig. 5b), IgM (trace), C3 (3-4+), and kappa (1-2+), and lambda (2-3+) light chains in a granular to confluent granular pattern, with scattered coarsely granular deposits. There was no specific glomerular staining for IgG or C1q, and no extraglomerular immune deposits were seen.

A preliminary diagnosis of an IgA-dominant, immune complex-mediated GN with mesangial and endocapillary hypercellularity and a rare cellular crescent, was conveyed to the patient's nephrologist. It was noted that the major differential diagnosis was IgA nephropathy versus IgA-dominant PIGN(infection-related) and that EM would be performed to resolve this differential diagnosis.

By EM (Fig. 5c, d), GBMs were globally thickened consistent with diabetic nephropathy, but otherwise normal in structure. Several capillary lumina contained leukocytes including monocytes and some neutrophils. Mesangial areas contained several, well-defined electron-dense 
Table 2. Differentiating IgA nephropathy and IgA-dominant PIGN

\begin{tabular}{lll}
\hline & IgA nephropathy & IgA dominant PIGN \\
\hline IF & IgA-dominant or codominant staining & $\begin{array}{l}\text { IgA-dominant immunoglobulin staining, with codominant } \\
\text { or stronger C3 staining }\end{array}$ \\
\hline IF pattern & Granular mesangial staining, variable capillary wall staining & $\begin{array}{l}\text { Granular to coarse granular mesangial and segmental } \\
\text { capillary wall staining }\end{array}$ \\
\hline EM & $\begin{array}{l}\text { Electron-dense mesangial } \pm \text { subendothelial deposits, rarely } \\
\text { subepithelial deposits }\end{array}$ & $\begin{array}{l}\text { Electron-dense and/or smudgy mesangial deposits, often } \\
\text { subepithelial "hump"-shaped deposits, often small } \\
\text { subendothelial deposits }\end{array}$ \\
\hline LM & $\begin{array}{l}\text { Segmental or global mesangial hypercellularity } \pm \text { endocapillary } \\
\text { hypercellularity, sometimes focal crescents }\end{array}$ & $\begin{array}{l}\text { Mesangial and endocapillary hypercellularity, intracapillary } \\
\text { neutrophils in acute phase, sometimes focal crescents }\end{array}$ \\
\hline $\begin{array}{l}\text { Serum complement } \\
\text { C3 level }\end{array}$ & Normal & \begin{tabular}{l} 
Low or normal \\
\hline
\end{tabular} \\
\hline
\end{tabular}

EM, electron microscopy; LM, light microscopy; IF, immunofluorescence; PIGN, postinfectious glomerulonephritis.

(immune complex) deposits subjacent to paramesangial basement membranes. Smudgy, irregular to rounded deposits were also noted centrally within a mildly expanded mesangial matrix. Small intramembranous and subendothelial deposits were also noted, some of which appeared mildly elongated and were very segmentally associated with early GBM duplication. There were prominent subepithelial "hump"-shaped deposits in mesangial waist regions between adjacent capillary loops, as well as along peripheral glomerular capillary walls (Fig. 5d). No TRIs were seen in the endothelial cell cytoplasm. Podocytes displayed moderately extensive but not complete foot process effacement. No tubular basement membrane deposits were seen.

Adiagnosis ofsubacuteIgA-dominantPIGN(infectionrelated) was made. Further discussion with the patient's clinical team revealed that several weeks prior, he had methicillin-sensitive Staphylococcus aureus bacteremia from an infected cardiac pacemaker.

\section{Discussion}

IgA-dominant PIGN was first characterized in 2003 [30] in a series of patients with underlying diabetic nephropathy. Renal biopsy revealed histologic features consistent with acute poststreptococcal GN, but with immune complex deposits containing IgA as the sole or dominant immunoglobulin by IF, mimicking IgA nephropathy. Each of the patients in this case series had underlying staphylococcal infections, suggestive of a different variant of acute PIGN. Since then, additional reports and case series have described the varying clinicopathologic features of IgA-dominant PIGN [31-35], including a predilection for older patients and frequent underlying comorbidities such as diabetes.

Differentiating between IgA nephropathy and IgAdominant PIGN (Table 2) is essential, due to differing treatments and prognoses. This is especially important where an infection may not be clinically apparent in a patient who is otherwise being biopsied for hematuria and proteinuria. Both entities are characterized by IgA-dominant staining by IF, which tends to be largely mesangial with variable capillary wall involvement. By LM, both IgA nephropathy and IgA-dominant PIGN can display mesangial and endocapillary hypercellularity, although comparative analyses have shown that glomerular neutrophilic infiltration is more common in IgA-dominant PIGN [32]. Similarly, crescent formation can be seen in both, although more commonly in IgA-dominant PIGN [33]. The presence of subepithelial "hump"-shaped deposits by EM is extremely helpful in differentiating between the 2 disease entities, although such deposits may not be present in all cases of IgA-dominant PIGN $[30,35]$. For the sake of completeness, it should also be mentioned that another mimicker of IgA-dominant PIGN could be IgA nephropathy superimposed on a resolving or largely resolved, nonIgA-dominant PIGN. In such cases it is helpful to closely examine the distribution of IF staining for IgA and C3 as the deposits tend to colocalize in IgA-dominant PIGN as part of the same pathogenic process. EM may also be helpful in discriminating between the 2 entities, particularly if the IgA nephropathy and infection-related GN are temporally distinct, with recent appearing mesangial immune complex deposits (IgA) contrasting with largely resorbed subepithelial/intramembranous "hump"-shaped deposits. 
Fig. 6. Immmunotactoid glomerulopathy. a PAS stain shows faintly PAS-positive intracapillary immune aggregates. The unaffected portions of the glomerulus have single contoured GBMs and patent capillary lumina without evidence of endocapillary hypercellularity or crescents (original magnification, $\times 200)$. b IF for kappa light chains reveals global mesangial and focal intracapillary (arrow) staining; there was similar staining for IgM without significant staining for IgG, IgA, and lambda light chains. c, d EM shows aggregates of tubular structures in mesangial, subendothelial, and intracapillary locations that often intersect with each other, occasionally at right angles. The microtubules are hollowcored and measure approximately $40 \mathrm{~nm}$ in diameter (uranyl acetate and lead citrate stain, original magnifications, $\times 7,000$ (c) and 14,000 (d)). EM, electron microscopy; IF, immunofluorescence; GBMs, glomerular basement membranes; PAS, periodic acid-Schiff.
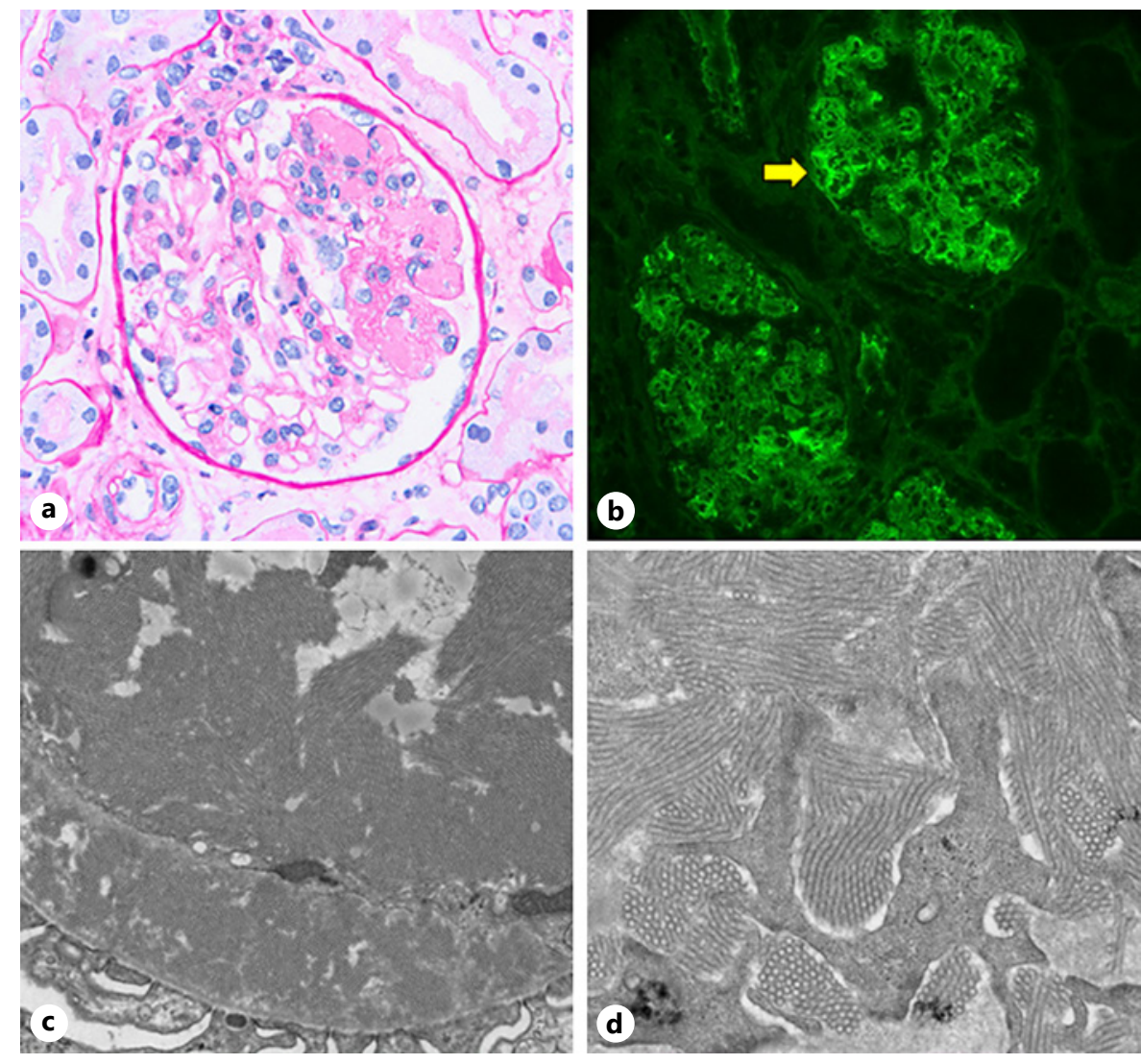

\section{Teaching Example \#5 - Immunotactoid Glomerulopathy}

A 72-year-old male patient, with a history of hypertension and Waldenstrom's macroglobulinemia, presented with nephrotic range proteinuria and AKI. Serum and urine protein electrophoresis and immunofixation revealed an IgM-kappa paraprotein. Testing for cryoglobulins was repeatedly negative.

Tissue processed for LM contained 25 glomeruli, 2 of which were globally sclerotic. Glomeruli were normal in size with predominantly single contoured capillary basement membranes. Some glomeruli (Fig. 6a) displayed near-occlusive intracapillary accumulation of modestly PAS-positive eosinophilic material which was fuchsinophilic on Masson' trichrome stain and negative on silver stains, most consistent with immune-type deposits. Similar deposits were seen in a subendothelial distribution, focally associated with GBM double contours. Several glomeruli also displayed segmental intracapillary infiltration by neutrophils and mononuclear leukocytes. Mesangial areas were frequently expanded by the immune de- posits, with segmental hypercellularity. No crescents or necrotizing lesions were present. Due to the history of paraproteinemia, a Congo red stain for amyloid was performed and was negative.

IF studies revealed glomerular capillary wall and mesangial staining for IgM and kappa light chains (Fig. 6b) in a granular to confluent granular pattern and global distribution, without significant staining for IgG, IgA, or lambda light chain. Several glomerular capillary lumina also contained focally occlusive aggregates that also stained for IgM and kappa only. Tubular casts stained for both kappa and lambda light chains in a polytypic pattern, with equal intensity. No staining of tubular basement membranes was noted.

EM (Fig. 6c, d) revealed several capillary lumina which appeared to be completely occluded by immune aggregates displaying a microtubular substructure composed of hollow-cored tubules measuring approximately $40 \mathrm{~nm}$ in diameter. The microtubules ranged in length from short to elongated, frequently forming streaming and long parallel arrays, bundles of which were often oriented perpendicular to each other. Similar microtubular material was exten- 
Table 3. Differential diagnosis of immune-mediated glomerular lesions with fibrillar/microtubular substructure

\begin{tabular}{|c|c|c|c|c|c|c|}
\hline & Special stains & Structure & $\begin{array}{l}\text { Diameter, } \\
\mathrm{nm}\end{array}$ & Orientation & $\begin{array}{l}\text { Most common glomerular } \\
\text { distribution }\end{array}$ & $\begin{array}{l}\text { Extraglomerular } \\
\text { involvement }\end{array}$ \\
\hline Amyloid & $\begin{array}{l}\text { Congo red, thioflavin T } \\
{[42]}\end{array}$ & Fibril & $8-12$ & $\begin{array}{l}\text { Random, } \\
\text { nonbranching }\end{array}$ & $\begin{array}{l}\text { Mesangium, subendothelial, } \\
\text { intramembranous, subepithelial }\end{array}$ & Yes \\
\hline FGN & $\begin{array}{l}\text { DNAJB9 } \\
\text { immunohistochemistry } \\
{[6]}\end{array}$ & Fibril & $16-24$ & $\begin{array}{l}\text { Random, } \\
\text { nonbranching }\end{array}$ & $\begin{array}{l}\text { Mesangium, subendothelial, } \\
\text { intramembranous, subepithelial }\end{array}$ & Rare \\
\hline ITG & & $\begin{array}{l}\text { Microtubule, } \\
\text { hollow core }\end{array}$ & $30-50$ & $\begin{array}{l}\text { Elongated, often } \\
\text { parallel arrays }\end{array}$ & $\begin{array}{l}\text { Mesangium, subendothelial, } \\
\text { intramembranous, subepithelial }\end{array}$ & Rare \\
\hline $\begin{array}{l}\text { Cryoglobulinic } \\
\text { glomerulonephritis }\end{array}$ & & $\begin{array}{l}\text { Cylindrical/annular; } \\
\text { hollow core }\end{array}$ & $30-35$ & $\begin{array}{l}\text { Short, randomly } \\
\text { oriented }\end{array}$ & $\begin{array}{l}\text { Subendothelial, capillary lumen, } \\
\text { mesangium }\end{array}$ & $\begin{array}{l}\text { Sometimes } \\
\text { (vascular) }\end{array}$ \\
\hline Lupus nephritis & & Fibrils/tubules & $\begin{array}{l}10-100 \\
{[43]}\end{array}$ & Fingerprint- like & $\begin{array}{l}\text { mesangial, subendothelial, and/or } \\
\text { subepithelial }\end{array}$ & Yes \\
\hline
\end{tabular}

FGN, fibrillary glomerulonephritis; ITG, immunotactoid glomerulopathy.

sively deposited in the mesangium, with segmental extension along the subendothelial aspect of adjacent capillary walls associated with GBM duplication and segmental mesangial cell interposition. Elsewhere, there were large and confluent subendothelial microtubular deposits which involved the entire circumference of involved capillary loops. No extraglomerular deposits or TRIs were identified. Podocyte foot processes were partially effaced. A diagnosis of immunotactoid glomerulopathy (ITG) was made.

\section{Discussion}

LM and IF revealed an immune-mediated glomerular lesion with multifocal intracapillary "immune pseudothrombi" and extensive mesangial and segmental subendothelial deposits that were IgM-kappa restricted. These findings were most suggestive of cryoglobulinemic GN related to a monoclonal (type I), IgM-kappa cryoglobulin. However, the ultrastructural study revealed findings typical of ITG.

The differential diagnosis of immune-mediated glomerular lesions displaying an organized substructure is outlined in Table 3. Although the intracapillary immune aggregates seen in this case are most often associated with cryoglobulinemic GN and are somewhat unusual for ITG, this pattern has been described in the latter [36]. The presence of large and confluent subendothelial deposits suggests that the occlusive intracapillary appearance of the microtubular structures may actually represent subendothelial deposits, which have impinged into the capillary luminal space, similar to what has been described in the "hyaline thrombi" of active lupus nephritis. The char- acteristic hollow-core, microtubular substructure of ITG, and the relatively larger cross-sectional diameters are key in distinguishing it from other deposits with a fibrillar substructure such as amyloidosis and fibrillary GN (FGN). Congo red positivity can also help establish a diagnosis of amyloidosis as ITG and FGN are almost exclusively Congo red-negative, with exceedingly rare exceptions [37]. Ultrastructurally, amyloid and FGN can also both demonstrate significant GBM involvement with subepithelial and transmembrane infiltration, which is not typically seen in ITG [38]. Unlike FGN, ITG also tends to be monoclonal or oligoclonal in the majority of cases [39], most often IgG-associated. The finding of an IgM paraprotein in this case is also somewhat atypical, although this has been reported [40].

In the largest of the ITG case series reported to date, a significant predisposition for an underlying lymphoplasmacytic disorder and monoclonal paraprotein was reported [41], as was seen in this case. However, it should be noted that examination of serial biopsies in ITG patients has also revealed evolution of IF findings from a polyclonal to a monoclonal process, sometimes spanning several years [39].

\section{Teaching Example \#6 - Collagen Type III Glomerulopathy}

A 59-year-old white man with a history of prediabetes presented with a sub-nephrotic range and slowly rising serum creatinine. He first reported mild proteinuria with 
Fig. 7. Collagen type III glomerulopathy. a By LM, an enlarged glomerulus shows global mesangial expansion by homogeneous, amorphous appearing, PAS-negative material that also fills capillary lumina, without endocapillary hypercellularity (periodic acid-Schiff stain; original magnification, $\times 200)$. b EM shows a subendothelial space expanded with fibrillar material. Glomerular capillary basement membranes themselves are devoid of the material (uranyl acetate and lead citrate stain, original magnification, $\times 7,200)$. c The fibrils are randomly arranged, curved, and banded (original magnification, $\times 14,000$ ). d Indirect IF for type III collagen. This case (different from the case study presented) showed deposition of fibrillar material predominantly in the mesangium. Type III collagen staining is strongly positive in the mesangium (arrows) and also in periglomerular interstitial fibrosis (arrowheads) (original magnification, $\times 400$ ). EM, electron microscopy; LM, light microscopy; IF, immunofluorescence; PAS, periodic acidSchiff.
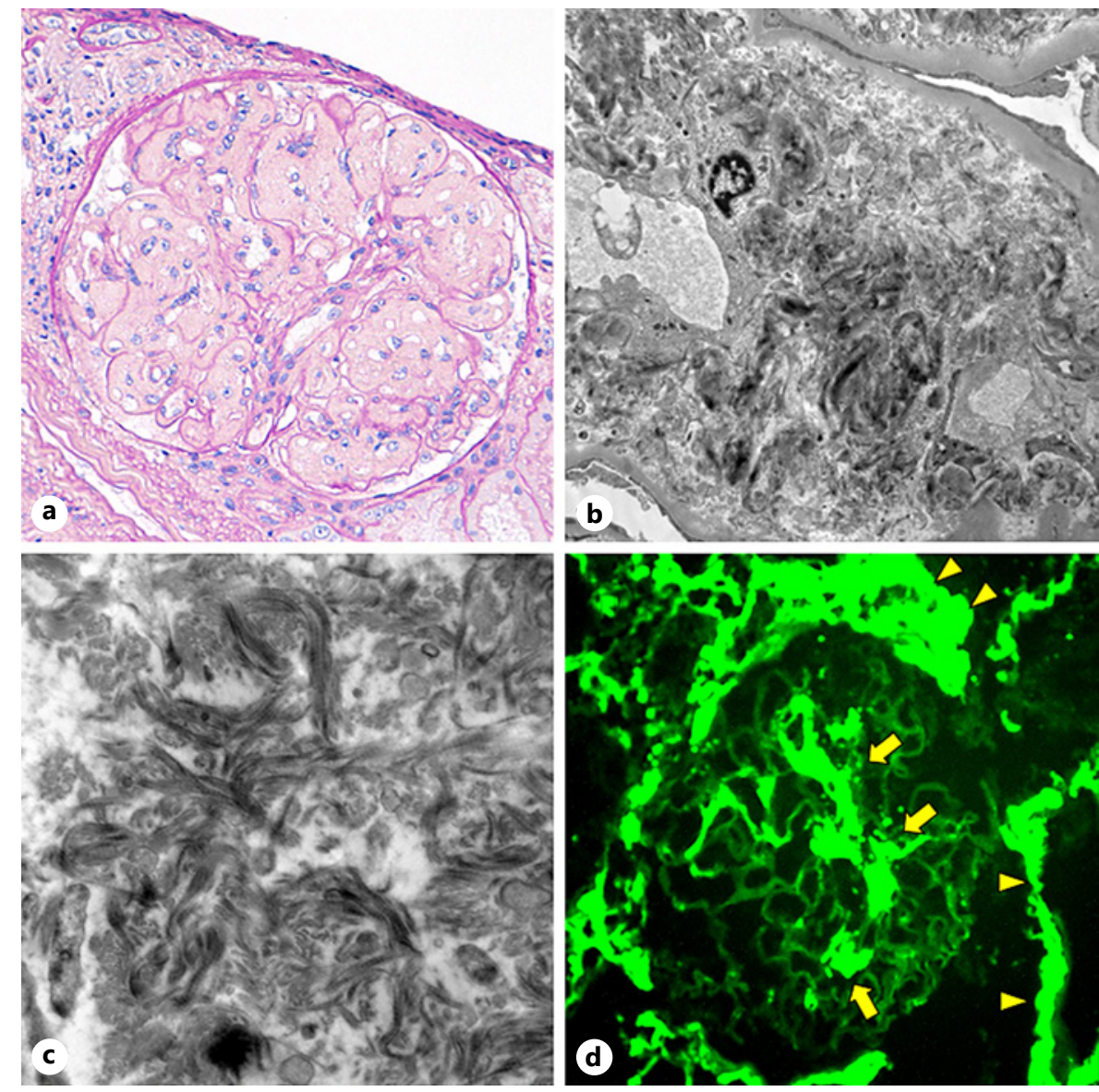

normal serum creatinine 8 years earlier. Proteinuria slowly increased over time to $2.5 \mathrm{~g} /$ day with a serum creatinine of $1.3 \mathrm{mg} / \mathrm{mL}$ (baseline $1.0 \mathrm{mg} / \mathrm{dL}$ ). Serologic workup was negative, and there was no family history of renal disease.

The portion of the renal biopsy processed for LM contained 38 glomeruli, of which 3 were globally sclerosed. The non-sclerosed glomeruli were normocellular and enlarged with lobular accentuation of the tufts, capillary wall thickening, and prominent mesangial expansion due to accumulation of homogeneous, amorphous-appearing eosinophilic material that was negative on periodic acidSchiff (PAS; Fig. 7a) and silver stains. A Congo red stain was negative for amyloid. There were segmental GBM double contours without spikes or craters. No crescents or necrotizing lesions were noted. Proximal tubules displayed evidence of injury with epithelial flattening and loss of brush borders. Approximately $20 \%$ of the sampled cortex was involved by tubular atrophy and interstitial fibrosis, which was associated with mild interstitial mono- nuclear cell inflammation. Arteries showed mild intimal fibrosis; arterioles showed mild hyalinosis. No vascular inflammation was identified. A Congo red stain was negative for amyloidosis.

Direct IF studies showed no glomerular deposits of immunoglobulins, complement components, or immunoglobulin light chains. EM showed prominent expansion of mesangial and subendothelial regions by curvilinear, banded fibrils which displayed periodicity of approximately $60 \mathrm{~nm}$, consistent with type III collagen glomerulopathy (Fig. 7b, c). The curvilinear fibrils did not permeate GBMs. No electron-dense (immune complex) deposits were identified nor were there powdery deposits or amyloid fibrils in any locations. There was extensive (approximately 80\%) podocyte foot process effacement.

Because of the ultrastructural findings, indirect IF for type III collagen was performed on the small amount of remaining IF tissue and showed segmental positive staining in the glomeruli. Taken together, a final diagnosis of collagen type III glomerulopathy was rendered. 


\section{Discussion}

Collagen type III glomerulopathy (also known as collagenofibrotic glomerulopathy, primary glomerular fibrosis, and collagen III glomerulopathy) is a rare idiopathic glomerular disease defined by abnormal accumulation of type III collagen in the mesangium and subendothelial space. Type III collagen is encoded by $\mathrm{Co}-$ $13 \mathrm{~A} 1$ gene located on chromosome $2 \mathrm{q} 32.2$ and is absent in the normal glomerulus [42, 43]. Earlier, some groups felt that type III collagen glomerulopathy represented a glomerular limited form of nail-patella syndrome; however, the familial occurrence of type III collagen glomerulopathy in some young patients suggests autosomal recessive inheritance, differing from the autosomal dominant pattern of the nail-patella syndrome [44]. In addition, while type III collagen glomerulopathy shows type III collagen deposition in the mesangium and subendothelial space that does not permeate the lamina densa of the GBM (Fig. 7c, d), nail-patella syndrome manifests type III collagen deposition within the GBMs.

The etiology and pathogenesis of type III collagen glomerulopathy remain unknown. It may be diagnosed at any age (reported range 3-79 years) [45, 46], with male and female individuals affected equally. Patients present with proteinuria, edema, microscopic hematuria, and hypertension, often with insidious progression to end-stage renal disease. Gubler et al. [44] also reported a frequent association of hemolytic uremic syndrome with type III collagen glomerulopathy in children, and one case was associated with factor $\mathrm{H}$ deficiency [47].

Histologically, type III collagen glomerulopathy typically shows enlarged glomeruli with prominent accumulation of amorphous material along capillary loops and in markedly expanded mesangial areas with absent or mild mesangial hypercellularity, although some cases show predominantly glomerular capillary loop or mesangial involvement. The amorphous material is negative or weakly positive on PAS and methenamine silver stains, and stains pale blue on the Masson's trichrome stain. Some cases are said to have a membranoproliferative GN pattern because of the lobular accentuation of the glomerular tufts and GBM double contours; however, endocapillary hypercellularity is typically lacking. There is no significant deposition of immunoglobulins, complement components, or light chains. The mesangial and subendothelial amorphous material are strongly positive for type III collagen (Fig. 7d); such staining is usually indicated by the characteristic ultrastructural findings, to confirm the diagnosis.

An ultrastructural study is absolutely essential to make a definitive diagnosis as LM findings can mimic other dis- eases including FGN. Mesangial areas and subendothelial spaces show fibrillar collagen deposits on a relatively electron lucent background (Fig. 7b). The collagen forms stacked bundles that show irregular, frayed edges and are typically curved (Fig. 7c), rather than straight as in normal interstitial type III collagen. A periodicity of approximately $60 \mathrm{~nm}$ is present, similar to normal type III collagen. Subepithelial and intramembranous collagen fiber deposition is not seen. Electron-dense deposits are absent, and podocyte foot processes are variably effaced. The lamina densa of GBMs is of normal appearance without localized intramembranous lucencies or areas of mottling.

\section{Conclusion}

These 6 teaching examples represent a small sample highlighting the continuing importance of EM as part of the diagnostic workup for medical renal biopsies. In some instances, such as case 3 , the diagnostic findings are revealed only by EM, which was absolutely crucial. Failure to perform ultrastructural studies in such cases would result in an incorrect diagnosis with likely clinical consequences. As such, we continue to strongly recommend that tissue for EM be taken and stored in an appropriate fixative and ultrastructural studies be performed for all native renal biopsies, as well as appropriate renal allograft biopsies as recommended by the Banff consortium [2].

\section{Statement of Ethics}

This review article contains several teaching examples composed of de-identified histological images and mock simple clinical information, and is therefore exempt from Ethical Committee approval.

\section{Conflict of Interest Statement}

The authors have no conflicts of interest to disclose.

\section{Funding Sources}

M.Y. is supported by NCATS UCLA CTSI KL2 grant (KL2TR001882) and Cedars-Sinai CTSI Clinical Scholar Grant.

\section{Author Contributions}

M.Y., M.Y.L., J.H., and K.Y.M.R. analyzed data and wrote the manuscript with support from M.H. 


\section{References}

1 Haas M. A reevaluation of routine electron microscopy in the examination of native renal biopsies. J Am Soc Nephrol. 1997;8:70-6.

2 Haas M, Sis B, Racusen L, Solez K, Glotz D, Colvin RB, et al. Banff 2013 meeting report: inclusion of C4d-negative antibody-mediated rejection and antibody-associated arterial lesions. Am J Transplant. 2014;14:272-83.

3 Haas M, Mirocha J. Early ultrastructural changes in renal allografts: correlation with antibody-mediated rejection and transplant glomerulopathy. Am J Transplant. 2011;11: 2123-31.

4 Beck LH Jr, Bonegio RG, Lambeau, Beck DM, Powell DW, Cummins TD, et al. Mtype phospholipase A2 receptor as target antigen in idiopathic membranous nephropathy. N Engl J Med. 2009;361:11-21.

5 Tomas NM, Beck LH Jr, Meyer-Schwesinger, Seitz-Polski B, Ma H, Zahner G, et al. Thrombospondin type-1 domain-containing 7A in idiopathic membranous nephropathy. N Engl J Med. 2014;371:2277-87.

6 Andeen NK, Yang HY, Dai D, MacCoss MJ, Smith KD. DnaJ homolog subfamily B member 9 is a putative autoantigen in fibrillary GN. J Am Soc Nephrol. 2018;29:231-9.

7 Jennette JC, Falk RJ. Adult minimal change glomerulopathy with acute renal failure. Am Jidney Dis. 1990;16:432-7.

8 Nephrotic syndrome in children: prediction of histopathology from clinical and laboratory characteristics at time of diagnosis. A report of the International Study of Kidney Disease in Children. Kidney Int. 1978;13. 159-65.

9 Waldman M, Crew RJ, Valeri, Busch J, Stokes B, Markowitz G, et al. Adult minimalchange disease: clinical characteristics, treatment, and outcomes. Clin J Am Soc Nephrol. 2007;2:445-53.

10 Vivarelli M, Massella L, Ruggiero B, Emma F. Minimal change disease. Clin J Am Soc Nephrol. 2017;12:332-45.

11 Ehrenreich T, Churg J. Pathology of membranous nephropathy. Pathol Annu. 1968;3: 145-86.

12 Jennette JC, Iskandar SS, Dalldorf FG. Pathologic differentiation between lupus and nonlupus membranous glomerulopathy. Kidney Int. 1983;24:377-85

13 Lai KN, Li PK, Lui S, Au TC, Tam JS, Tong $\mathrm{KL}$, et al. Membranous nephropathy related to hepatitis B virus in adults. N Engl J Med. 1991;324:1457-63.

14 Latt N, Alachkar N, Gurakar A. Hepatitis C virus and its renal manifestations: a review and update. Gastroenterol Hepatol. 2012;8: 434-45.

15 Glassock RJ. Secondary membranous glomerulonephritis. Nephrol Dial Transplant. 1992;7(Suppl 1):64-71.
16 Markowitz GS. Membranous glomerulopathy: emphasis on secondary forms and disease variants. Adv Anat Pathol. 2001;8:11925.

17 Haas M. Alport syndrome and thin glomerular basement membrane nephropathy: a practical approach to diagnosis. Arch Pathol Lab Med. 2009; 133:224-32.

18 Kashtan CE. Alport syndrome and thin glomerular basement membrane disease. J Am Soc Nephrol. 1998;9(9):1736-50.

19 Gubler MC, Heidet L, Antignac C. Alport syndrome, familial benign hematuria, nailpatella syndrome, type III collagen glomerulopathy, and Pierson syndrome. 7th ed. In: Jennette JC, Silva FG, Olson JL, D’Agati VD, editors. Heptinstall's pathology of the kidney. Philadelphia, PA: Wolters Kluver; 2015. Vol. 1; p. 525-58.

20 Hinglais N, Grünfeld JP, Bois E. Characteristic ultrastructural lesion of the glomerular basement membrane in progressive hereditary nephritis (Alport's syndrome). Lab Invest. 1972;27:473-87.

21 Rumpelt HJ. Hereditary nephropathy (Alport syndrome): correlation of clinical data with glomerular basement membrane alterations. Clin Nephrol. 1980;13:203-7.

22 Heidet L, Gubler MC. The renal lesions of Alport syndrome. J Am Soc Nephrol. 2009; 20:1210-5

23 Mazzucco G, Barsotti P, Muda AO, Fortunato $\mathrm{M}$, Mihatsch $\mathrm{M}$, Torri-Tarelli $\mathrm{L}$, et al. Ultrastructural and immunohistochemical findings in Alport's syndrome: a study of 108 patients from 97 Italian families with particular emphasis on COL4A5 gene mutation correlations. J Am Soc Nephrol. 1998;9: 1023-31.

24 Arrondel C, Deschênes G, Le Meur Y, Viau A, Cordonnier C, Fournier A, et al. A large tandem duplication within the COL4A5 gene is responsible for the high prevalence of Alport syndrome in French Polynesia. Kidney Int. 2004;65:2030-40.

25 Hill GS, Jenis EH, Goodloe S Jr. The nonspecificity of the ultrastructural alterations in hereditary nephritis with additional observations on benign familial hematuria. Lab Invest. 1974;31:516-32.

26 Nadasdy T, Abdi R, Pitha J, Slakey D, Racusen L. Diffuse glomerular basement membrane lamellation in renal allografts from pediatric donors to adult recipients. Am J Surg Pathol. 1999;23:437-42.

27 Akhtar M, Al-Sabban E. Alport-like glomerular changes in a patient with nephrotic syndrome: report of a case. Pediatr Nephrol. 2000;14:973-5.

28 Chevalier C, Colon S, Faraj G, Bouvier R, Pinçon JA, Cochat P. [Glomerulopathy and hypomelanosis of Ito]. Nephrologie. 1997; 18:125-7.
29 Ito S, Hataya H, Ikeda M, Takata A, Kikuchi $\mathrm{H}$, Hata J, et al. Alport syndrome-like basement membrane changes in Frasier syndrome: an electron microscopy study. Am J Kidney Dis. 2003;41:1110-5.

30 Nasr SH, Markowitz GS, Whelan J, Albanese JJ, Rosen RM, Fein DA, et al. IgA-dominant acute poststaphylococcal glomerulonephritis complicating diabetic nephropathy. Hum Pathol. 2003;34:1235-41.

31 Haas M, Racusen LC, Bagnasco SM. IgAdominant postinfectious glomerulonephritis: a report of 13 cases with common ultrastructural features. Hum Pathol. 2008;39: 1309-16.

32 Koo TY, Kim GH, Park MH. Clinicopathologic features of IgA-dominant postinfectious glomerulonephritis. Korean J Pathol. 2012;46:105-14.

33 Wen YK, Chen ML. Discrimination between postinfectious IgA-dominant glomerulonephritis and idiopathic IgA nephropathy. Ren Fail. 2010;32:572-7.

34 Nasr SH, D’Agati VD. IgA-dominant postinfectious glomerulonephritis: a new twist on an old disease. Nephron Clin Pract. 2011; 119:c18-25.

35 Satoskar AA, Suleiman S, Ayoub, Hemminger J, Parikh S, Brodsky SV, et al. Staphylococcus infection-associated GN - spectrum of IgA staining and prevalence of ANCA in a single-center cohort. Clin J Am Soc Nephrol. 2016;12:39-49.

36 Schwartz MM, Korbet SM, Lewis EJ. Immunotactoid glomerulopathy. J Am Soc Nephrol. 2002;13:1390-7.

37 Alexander MP, Dasari S, Vrana JA, Riopel J, Valeri AM, Markowitz GS, et al. Congophilic fibrillary glomerulonephritis: a case series. Am J Kidney Dis. 2018;72:325-36.

38 Fogo A, Qureshi N, Horn RG. Morphologic and clinical features of fibrillary glomerulonephritis versus immunotactoid glomerulopathy. Am J Kidney Dis. 1993;22:367-77.

39 Carrara C, Ferucci E, Emili S, Toukatly MN, Nicosia RF, Alpers CE. Immunotactoid glomerulopathy of 10-years' duration: insights gained from sequential biopsies. Kidney Int Rep. 2017;2:978-83.

40 Da'as N, Kleinman Y, Polliack A, Amir G, Ne'eman Z, Kopolovic J, et al. Immunotactoid glomerulopathy with massive bone marrow deposits in a patient with IgM kappa monoclonal gammopathy and hypocomplementemia. Am J Kidney Dis. 2001;38:395-9.

41 Nasr SH, Fidler ME, Cornell LD, Leung N, Cosio FG, Sheikh SS, et al. Immunotactoid glomerulopathy: clinicopathologic and proteomic study. Nephrol Dial Transplant. 2012;27:4137-46. 
42 Emanuel BS, Cannizzaro LA, Seyer JM, Myers JC. Human alpha 1(III) and alpha 2(V) procollagen genes are located on the long arm of chromosome 2. Proc Natl Acad Sci U S A. 1985;82:3385-9.

43 Morita H, Hasegawa T, Minamoto, Oda Y, Inui $\mathrm{K}$, Tayama $\mathrm{H}$, et al. Collagenofibrotic glomerulopathy with a widespread expression of type-V collagen. Virchows Arch. 2003;442:163-8.
44 Gubler MC, Dommergues JP, Foulard, Bensman A, Leroy JP, Broyer M, et al. Collagen type III glomerulopathy: a new type of hereditary nephropathy. Pediatr Nephrol. 1993;7:354-60.

45 Pizzo HP, Haas M, Puliyanda D. Collagen type III glomerulopathy. Kidney Int. 2018; 93:1490.
46 Fukami K, Yamagishi S, Minezaki T, Nishi S, Hisano S, Okuda S. First reported case of collagenofibrotic glomerulopathy with a fullhouse pattern of immune deposits. Clin Nephrol. 2014;81:290-5.

47 Vogt BA, Wyatt RJ, Burke BA, Simonton SC, Kashtan CE. Inherited factor $\mathrm{H}$ deficiency and collagen type III glomerulopathy. Pediatr Nephrol. 1995;9:11-5. 